$$
\text { **** Nur für den internen Gebrauch ***** }
$$

Sebastian Büttner | Thomas Laux [Hrsg.]

\title{
Umstrittene Expertise
}

Zur Wissensproblematik der Politik

Leviathan Sonderband 38 | 2021

\section{Nomos}


Onlineversion

Nomos eLibrary

Die Deutsche Nationalbibliothek verzeichnet diese Publikation in der Deutschen Nationalbibliografie; detaillierte bibliografische Daten sind im Internet über http://dnb.d-nb.de abrufbar.

ISBN 978-3-8487-8284-0 (Print)

ISBN 978-3-7489-1141-8 (ePDF)

1. Auflage 2021

(c) Nomos Verlagsgesellschaft, Baden-Baden 2021. Gesamtverantwortung für Druck und Herstellung bei der Nomos Verlagsgesellschaft mbH \& Co. KG. Alle Rechte, auch die des Nachdrucks von Auszügen, der fotomechanischen Wiedergabe und der Übersetzung, vorbehalten. Gedruckt auf alterungsbeständigem Papier. 


\section{Leviathan}

Jahrgang $49 \cdot$ Sonderband $38 \cdot 2021$

Inhaltsübersicht

Vorwort

\section{Einführung}

Sebastian M. Büttner und Thomas Laux

Umstrittene Expertise und die Wissensproblematik der Politik: Eine Einführung

\section{Ambivalenzen von Expertise}

Roland Czada

Zwischen Sachzwangideologie und Technokratiekritik

Diskurskapriolen um Technikbewertungen und Expertenrollen

Holger Straßheim

Paradoxien und Pendel: Umstrittene Expertise in der Weltgesellschaft

Nils C. Kumkar

Unbestimmte Negation: Gegenexpertise als alternative Fakten

\section{Das Spannungsfeld von wissenschaftlicher Evidenz und Politik}

Eva Barlösius und Eva Ruffing

Die Infragestellung wissenschaftlichen Wissens und wissenschaftlicher Expertise

Eine sozialwissenschaftliche Heuristik

Johannes Schmoldt

Das Wissen der Urteilskraft

Politisches Handeln und Entscheiden im Spannungsfeld von Erfahrung und

Evidenz

\section{Krisenexpertise: Umstrittene Problemlösungen}

Caspar Hirschi

Expertise in der Krise

Zur Totalisierung der Expertenrolle in der Euro-, Klima- und Coronakrise 
Silke Beck und Julian Nardmann

Wissenschaftliche Rückendeckung für politische Alternativlosigkeit?

Kontroversen um Expertisen in der deutschen Corona-Politik ....

\section{Umstrittene Politikberatung: Expertise in der Kritik}

Jasmin Theres Beneke und Marian Döhler

»Mehr Berater als Beamte«?

Eine empirische Analyse zur externen Beratung der Bundesministerien

Stephan Pühringer

Zur Pluralität der ökonomischen Politikberatung in Deutschland

Eine empirische Untersuchung

\section{Umstrittene Expertise in der Klimapolitik: Über die Politisierung des Wissens}

Thomas Laux

Vom Protest zur Produktion von Expertise

Die Umweltbewegung und die Herausbildung eines Feldes der Expertise zur Umwelt-

und Energiepolitik in Deutschland

Thomas Kern und Dabla Opitz

Disruptive Wissenschaft? Die Mobilisierung von Fridays for Future in den USA

und Deutschland

Fabian Hempel

Expertenwissen als Kippelement ökologischer Ignoranz?

Ein literatursoziologischer Blick auf den Status wissenschaftlicher Expertisen in der

Klimapolitik

\section{Die globale Dimension von Expertise: Strukturen und Ambivalenzen}

Luca Tratschin

Katastrophenkommunikation, holistische Perspektiven und die Expansion von

Expertise

Welt- und Gesellschaftsentwürfe im Spiegel zukünftiger Pandemien

Alejandro Esguerra

Zur politischen Epistemologie des Anthropozäns: Epistemische Autorität und institutionelles Design globaler Expertenorganisationen

Leopold Ringel, Julian Hamann und Jelena Brankovic

Unfreiwillige Komplizenschaft: Wie wissenschaftliche Kritik zur Beharrungskraft von

Hochschulrankings beiträgt 


\section{Forum: Reflexionen zur Corona-Pandemie}

Richard Münch

Benevolenter Paternalismus: Regieren nach SARS-CoV-2

Michaela Pfadenhauer

Erforderliche Expertise oder Herrschaft der grauen Herren?

Eine professionssoziologische Perspektive auf Expertenwissen zu Beginn der Corona-

Pandemie

Klaus Ferdinand Gärditz

Wissenschaftliche Rationalität, politische Willensbildung und rechtlich-institutionelle Wissensverantwortung

Die Pandemie als Anschauungsfall

Johannes Pantenburg und Benedikt Sepp

Wissen, hausgemacht. Selbstverständnis, Expertisen und Hausverstand der

'Querdenker

Andreas Knie und Dagmar Simon

Doppelte Validierung von Expertise: Wissenschaftliche Politikberatung als

Co-Production 
Caspar Hirschi

\title{
Expertise in der Krise
}

\section{Zur Totalisierung der Expertenrolle in der Euro-, Klima- und Coronakrise}

\begin{abstract}
The scientific enterprise has a notable shortage of Honest Brokers of Policy Alternatives, with many scientists instead choosing to engage policy and politics as Issue Advocates, or more troubling for the sustainability of the scientific enterprise, as Stealth Issue Advocates. Roger A. Pielke Jr., The Honest Broker, 2007
\end{abstract}

\section{Komplexe Krisen und die Unmöglichkeit von «Tornado Politics»}

Krisen sind eine Frage der Wahrnehmung. Sie entstehen, wenn sich der Erwartungshorizont, den eine Gesellschaft vom normalen Gang der Dinge hat, durch den Einbruch besorgniserregender Eindrücke verdunkelt. Heute empfangen die meisten Menschen die ersten Störsignale ihrer Normalitätserwartung durch mediale Vermittlung. In aller Regel sind es auch Medien, welche diese Signale zu Krisendiagnosen verdichten, bevor sie sich in der Lebenswirklichkeit der Betroffenen bemerkbar machen - wenn sie das überhaupt tun.

Es gibt Krisen, die nie den Eskalationssprung vom Medienereignis zum unmittelbar erlebten Verhängnis machen. Nach einer Zeit der medial geschürten Angst und bangen Erwartung lösen sie sich in Luft auf. Dann gibt es Krisen, in denen die medial vermittelte und die alltäglich erlebte Wahrnehmung zwangsläufig auseinanderfallen. Die Bedrohung bleibt entweder abstrakt, weil sie rechtzeitig abgewendet oder frühzeitig antizipiert wird, oder sie tritt aufgrund der Nebenwirkungen ihrer Bekämpfung in den Hintergrund. Solche Einschnitte können als komplexe Krisen bezeichnet werden, wobei sich «Komplexität» nicht auf ihre Ursachen, sondern auf ihre verschiedenen Wahrnehmungsebenen bezieht. In demokratischen Gesellschaften bieten komplexe Krisen Stoff für endlose Kontroversen, angefangen mit divergierenden Vorstellungen von «Normalität» bis zu Fundamentaldifferenzen über die Existenz der Bedrohung selbst. Entsprechend ist der Weg in die Krise gepflastert mit Deutungskämpfen bei unsicherer Erkenntnislage, hohem Zeitdruck und widerstreitenden Handlungsgeboten. In liberalen Demokratien werden diese Deutungskämpfe öffentlich ausgetragen, und Krisen sind nur solange soziale Tatsachen, wie sich ihre Diagnostiker gegen konträre Wirklichkeitsentwürfe behaupten können.

Hier kommt die spezifische Krisenfunktion von Experten ${ }^{1}$ zum Tragen: Sie sind zu Dominatoren dieser Deutungskämpfe aufgestiegen. Ihre Präsenz in medialen

1 Der vorliegende Aufsatz bedient sich des generischen Maskulinums. Wenn weibliche Pluralformen gesetzt werden, sind Frauen gemeint. Das Vorgehen hat pragmatische und programmatische Gründe. Pragmatisch geht es darum, eine Sprache zu verwenden, mit der dieser Text auch in Zukunft lesbar bleibt. «Geschlechtergerechtes» Schreiben

Leviathan, 49. Jg., Sonderband 38/2021, S. 161-186 
Gefechten um die richtige Krisenpolitik ist ein junges Phänomen, im Unterschied zu ihrer Rolle als Krisenberater von politischen Exekutiven. Ausschlaggebend für die Medialisierung der Expertenrolle ist eine Entwicklung, die Alexander Bogner die «Epistemisierung des Politischen» nennt. ${ }^{2}$ Politischer Streit wird, angetrieben von der Wertsteigerung der Währung "Wissen», immer mehr im Modus des Wissenskonfliktes ausgetragen. Damit verbunden ist die Erwartung, Kontroversen durch eine Versachlichung politischer Entscheidungsprozesse vermeiden zu können. Wer sich politisch durchsetzen will, muss sich mit wissenschaftlicher Expertise aufrüsten. Die Epistemisierung des Politischen geht einher mit einer «Demokratisierung des Expertenwissens», wie Peter Weingart 2001 die Tendenz zum medialen Einsatz von Experten für alle möglichen Parteien und Positionen beschrieb. ${ }^{3}$ Die Hoffnung auf eine Versachlichung des politischen Streits hat sich nicht erfüllt. Stattdessen trieb die Medialisierung der Expertenrolle, die sich vom Irakkrieg bis zur Coronapandemie durch die jüngste Krisengeschichte zieht, die Polarisierung der demokratischen Streitkultur voran. Politische Gegner verwandeln sich unter den Vorzeichen eines Wissenskonflikts entweder in Realitätsverweigerer, die von falschen Experten verführt worden sind, oder in Betrüger, die sich die Dienste korrupter Experten gesichert haben. Ob man Menschen, die gegen die staatliche Seuchenpolitik protestieren, als «Coronaleugner» oder als «Maßnahmenkritiker» bezeichnet, ist für den Grad an Dissenstoleranz wesentlich.

Laut Roger Pielke kann wissenschaftliche Expertise in demokratischen Gesellschaften nur in bestimmten Ausnahmesituationen konsensbildend wirken. Die Ungewissheit über das, was ist, muss klein und der Wertekonsens über das, was sein soll, muss groß sein. Pielke bezeichnet die idealtypische Reaktion auf solche Situationen als «Tornado Politics». ${ }^{4}$ Man kennt die Gefahr, ist sich über das Ziel einer Krisenintervention einig und folgt den Anweisungen bewährter Fachleute, um den Schaden einzudämmen. In komplexen Krisen ist keines der beiden Kriterien für Tornadopolitik erfüllt. Werden hier Deutungskämpfe im Gewande von Wissenskonflikten ausgetragen, ist die Versuchung für involvierte

ist dafür am wenigsten geeignet, weil es laufend neue Ausdrucksformen erhalten muss, um dem progressiven Ideal einer immer perfekteren Inklusion durch symbolische Zeichen zu genügen. Forschungspublikationen, in denen die gerade geltende Moral stark auf die Sprache durchschlägt, altern schlecht; die Geschichte bietet dafür reichlich Anschauungsmaterial. Der programmatische Grund ist, dass ich die Wissenschaftssprache für das falsche Mittel halte, um inklusionspolitische Anliegen zu verfolgen. Sie wird durch «geschlechtergerechtes» Schreiben noch mehr zu einem Distinktionsinstrument, das akademisch schwach Sozialisierte, die ihre neusten Formeln nicht beherrschen, abwertet und ausschließt. Für die akademische Inklusion und Diversität sind universitäre Ämter und Berufungskommission da. Entsprechend setze ich mich als Dekan und Leiter von Berufungskommissionen dafür ein, die Vielfalt der geschlechtlichen, kulturellen, sozialen und ideologischen Hintergründe des Forschungspersonals unter Wahrung höchster wissenschaftlicher Qualitätskriterien zu fördern.

2 Bogner 2021, S. 122-124.

3 Weingart 2001, S. 161.

4 Pielke 2007, S. 41-42. 
Experten groß, epistemische Unsicherheiten kleinzureden, divergierende Werteeinstellungen auszublenden, und sich mit Voraussagen und Empfehlungen weiter vorzuwagen, als es der eigene Erkenntnisstand erlaubt. Die eigene Rolle soll dabei Unvereinbares vereinen. Man stellt sich gleichzeitig über die Niederungen des politischen Gezänks, indem man sich auf die alleinige Autorität der Wissenschaft beruft und greift in die Politik ein, um den Spielraum an Handlungsoptionen im Sinne der eigenen Werteprioritäten einzuschränken. So werden Experten, um einen weiteren Begriff von Pielke aufzugreifen, zu «Stealth Issue Advocates», den verdeckten Vorkämpfern normativer Anliegen. Diese aktivistische Form von Expertise kommt für Pielke dem Versuch gleich zu schwimmen, ohne nass zu werden. ${ }^{5}$ Geschieht dann doch das Unvermeidliche und Experten geraten in politische Kontroversen, können sie die Doppelrolle nur weiterspielen, wenn sie ihren Kritikern die wissenschaftliche Qualifikation absprechen, sich an der Diskussion zu beteiligen.

Der vorliegende Aufsatz stellt die These auf, dass die jüngste Kaskade an komplexen Krisen einer epistemischen Entgrenzung und normativen Totalisierung der Expertenrolle Vorschub geleistet hat, die historisch präzedenzlos, politisch riskant und demokratisch problematisch ist. Sie äussert sich in der Rollenkumulation einer medialen «Expertenprominenz», deren Vertreter zugleich als Intellektuelle, Berater und Aktivisten auftreten. Experten häufen mit ihr eine neue, von Widersprüchen geprägte Machtfülle an, die eine verfahrensgeleitete Politikberatung erschwert und die demokratische Diskussion polarisiert. Die Politik treibt diesen Prozess mit an, indem sie die mediale Unterstützung prominenter Experten in Anspruch nimmt, um geplante Maßnahmen vor der Beschlussfassung als wissenschaftliche Sachzwänge auszuweisen. Die mediale Expertenprominenz erzeugt zu Legitimationszwecken den Eindruck einer vermeintlichen Eindeutigkeit, die es in komplexen Krisen kaum gibt. ${ }^{6}$ Wie eine vergleichende Analyse der drei Krisen zeigt, verstellt die Medienfigur des totalisierten Experten auch den Blick für die spezifischen Ausprägungen des Zusammenspiels von Wissenschaft und Politik, das in jeder komplexen Krise aus situativen und systemischen Gründen verschieden organisiert ist.

\section{Expertokratische und populistische Illusion der Expertise}

Die jüngsten Krisen haben Prozesse verstärkt und beschleunigt, die seit längerem mit der Epistemisierung des Politischen in demokratischen Staaten verbunden sind. Es ist daher wichtig, die Grundtendenzen dieser Entwicklung zu beschreiben, bevor der Einsatz von Experten in den komplexen Krisen der jüngsten Zeit in den Blick genommen wird. Als Ausgangspunkt dafür bietet sich ein Aufsatz von Sheila Jasanoff aus dem Jahr 2003 an. In »(No?) Accounting for Expertise " konstatiert sie einen «unkritischen und theoretisch uninformierten Diskurs über

5 ebd. S. 7.

6 Scharpf 1999, S. 16-28.

Leviathan, 49. Jg., Sonderband 38/2021 
Expertise», der auf Seiten politischer Entscheidungsträger eine «instrumentelle Einstellung» zu Experten gefördert und in der Bevölkerung die Forderung nach einer öffentlicher «Rechenschaftspflicht» von Experten geschwächt habe. ${ }^{7}$ Laut Jasanoff koexistieren in demokratischen Entscheidungsprozessen zwei unvereinbare Sichtweisen auf Experten: Sie nennt diese das «elitistische» und das «relativistische» Modell. Das elitistische Modell schreibt Experten aufgrund ihres überlegenen Sachwissens einen privilegierten Einfluss auf Entscheidungsträger zu, der bis zum Anspruch auf verbindliche Umsetzung ihrer Empfehlungen reicht. Das relativistische Modell dagegen spricht ihnen diesen Einfluss aufgrund der Beschränktheit und Vorläufigkeit ihres Spezialwissens gerade ab und reduziert ihre Empfehlungen auf eine relevante Meinung unter vielen.

Die beiden Modelle sind im politischen Alltag deshalb attraktiv, weil sie sich je nach politischer Opportunität flexibel anwenden lassen, nicht selten durch dieselben Akteure. In Jasanoffs Verständnis stehen die Modelle also weniger für ideologische als für situative Positionierungen, die in der Politik jene «instrumentelle Einstellung» fördern, mit der Expertise als Mittel zur Erreichung politischer Ziele eingesetzt wird. Regierungen orientieren sich am relativistischen Modell, wenn sie Experten für die wissenschaftliche Untermauerung geplanter Maßnahmen rekrutieren, und sie bemühen das elitistische Modell, wenn sie die gleichen Experten für die öffentliche Rechtfertigung der Maßnahmen einsetzen. Dieses legitimatorische Vorgehen bietet sich besonders für politische Strategiewechsel an, die einen erheblichen Rechtfertigungsbedarf nach sich ziehen. ${ }^{8}$ Lässt sich eine kontroverse Entscheidung unter Berufung auf Experten als wissenschaftlich erforderlich darstellen, muss Widerspruch aus der Opposition zugleich die Regierung als auch die Wissenschaft in Frage stellen. Um dieser Zwickmühle zu entgehen, bleibt oppositionellen Parteien oft nur die Option übrig, ihrerseits das relativistische Modell zu bemühen und Gegenexperten aufzubieten, die dem politischen Streit einen wissenschaftlichen Dissens unterlegen.

Jasanoff kritisierte schon 2003, dass beide Modelle die Realität wissenschaftlicher Expertise in Demokratien schlecht abbilden. Was sie damals aber noch nicht ahnen konnte, war der markante Funktionswandel, den die Modelle mit der zunehmenden Polarisierung der Politik durchlaufen würden. Ihr instrumenteller Einsatz durch Politiker, die beide Modelle je nach Situation flexibel einsetzten, wurde überlagert vom ideologischen Bemühen, sie zu polaren Gegensätzen in einem Grabenkampf umzufunktionieren, den das eine Lager im Namen der Wissenschaft und das andere im Namen das Volkes führt. Im Zuge dieses Prozesses verhärtete sich das elitistische Modell zur expertokratischen Illusion, Aufgabe der Politik sei es, die Vorgaben der Wissenschaft umzusetzen, während das relativisti-

7 Jasanoff 2003; die folgenden Passagen sind in Teilen schon als gekürzter Vorabdruck dieses Aufsatzes in Hirschi 2021a und Hirschi 2021b erschienen.

8 Bekannte Beispiele sind der Atomausstieg der Regierung Merkel 2011 oder das Atomabkommen der Regierung Obama (und weiterer Nato-Staaten) mit dem Iran 2015. Hirschi 2018, S. 16; 297. 
sche Modell zur populistischen Illusion gerann, die Politik habe sich im Namen des Volkes und dessen Wahrheit gegen die Tyrannei der Experten zu wehren.

In aller Deutlichkeit entfaltete sich dieser Grabenkampf erstmals 2016 - und das gleich in zwei kapitalen Schlachten: dem britischen Brexit-Referendum und den amerikanischen Präsidentschaftswahlen. Neu war auch, dass in beiden Auseinandersetzungen jene Seite gewann, die den Wissenskonflikt mit pointierter Expertenfeindlichkeit fortsetzte und Wissenschaft, zumindest in politisch relevanten Belangen, als Quelle der Unwahrheit denunzierte. ${ }^{9}$ Damit zerbrach die bis dahin vorherrschende Annahme quer durchs politische Meinungsspektrum hindurch, es zahle sich in der demokratischen Politik aus, die wissenschaftliche Expertise auf der eigenen Seite zu haben. Der expertokratischen Illusion tat dies jedoch keinen Abbruch; im Gegenteil, sie erhielt nochmals mächtig Auftrieb. Wissenschaftliche Autoren landeten Bestseller mit Absagen an die Demokratie und Abgesängen auf die Expertise, womit sie ihrerseits einen Beitrag zur Polarisierungsdynamik im Zusammenhang mit Krisendiskursen leisteten. ${ }^{10}$

\section{Von schleichend zu akut: engagierte Expertise in der Klimakrise}

Als eigentliches Versuchslabor für den polarisierten Expertendiskurs diente jedoch weder der Brexit noch Trump, sondern die Klimapolitik. Hier ging es um ein politisches Handlungsfeld, das zwangsläufig von der Wissenschaft bestellt worden war, weil die Gefahr erst durch ihre Modelle sichtbar wurde. Die maßgeblichen Erkenntnisse lagen spätestens Mitte der neunziger Jahre vor, als der Weltklimarat konstatierte, dass «die Summe der Evidenz» einen «erkennbaren menschlichen Einfluss» auf die Klimaerwärmung nahelege. ${ }^{11}$ Seither wurde der Spielraum für die Inszenierung einer glaubwürdigen Gegenexpertise immer kleiner. Dennoch blieben die klimapolitischen Verwerfungen im Modus des Wissenskonfliktes gefangen. Alle Seiten beriefen sich auf überlegene Kenntnisse, wobei die Auffassungen, was als «Wissen» gelten darf, immer weiter auseinander drifteten. Gegner einer aktiven Klimapolitik hatten sich lange nach dem Vorbild der amerikanischen Tabak-Lobby auf möglichst renommierte Forscher berufen, die sich als "organisierte Skeptiker» im Sinne Robert Mertons ausgaben, um Zweifel am anthropogenen Treibhauseffekt zu säen. ${ }^{12}$ Als diese Taktik aufgrund des wachsenden Forschungskonsenses nicht mehr aufging, lancierten sie vermehrt direkte Angriffe auf die Glaubwürdigkeit führender Klimaexperten. Eine lose Koalition aus Hackern und Lobbyisten verunglimpfte Autoren des Weltklimarats nach 2009 im Zuge der "Climategate»-Kampagne als Manipulatoren wissenschaftlicher Befunde. Dadurch erhielten wiederum Verschwörungstheoretiker Auftrieb, die im

9 Eyal 2019, Kap. 1.

10 Brennan 2016; Nichols 2017; zur Kritik an Nichols siehe Hirschi 2018, S. 11-12, sowie Eyal 2019, Kap. 5.

11 Beck 2010, S. 297.

12 Oreskes und Conway 2010, S. 169; Merton 1942.

Leviathan, 49. Jg., Sonderband 38/2021 
Klimawandel einen organisierten Schwindel sahen, mit dem Klimaforscher eine expertokratische Weltherrschaft durch den Weltklimarat anstrebten. Gegen die wissenschaftlichen Daten und Modelle wurde das «höhere» Wissen über eine globale Verschwörung gegen die kleinen Leute in Stellung gebracht.

Die Klimawissenschaften reagierten auf die pauschale Infragestellung ihrer Expertise, indem sie sich jeder Diskussion verweigerten, den Bereich des als gesichert ausgeflaggten Wissens ausweiteten und den Grad des Forschungskonsenses mit neuen Methoden vorrechneten. Der Wissenskonflikt wurde indirekt fortgesetzt, als Kampf um die öffentliche Deutungshoheit unter Vermeidung einer Konfrontation. Es galt, Gegnern einer Präventionspolitik keine offenen Flanken für Skandalisierungsversuche zu bieten und jeden Anspruch auf argumentative Satisfaktionsfähigkeit zu verweigern. Je weniger die menschengemachte Erderwärmung der Vergangenheit in Zweifel stand, desto mehr rückte der zu erwartende Temperaturanstieg der Zukunft in den Blick. Dabei verhärteten sich Extrapolationen von Messreihen und Modellierungen von Zukunftsszenarien spätestens in der massenmedialen Kommunikation zu wissenschaftlichen Tatsachen.

Die mediale Präsentation von Modellen als Fakten wird dadurch begünstigt, dass Klimaforscher auch untereinander öffentliche Diskussionen über Ungewissheiten zu den künftigen Auswirkungen des Klimawandels vermeiden. Stattdessen heben sie nach außen den hohen Grad an Gewissheit in politisch relevanten Punkten hervor. Gewissheit erscheint dabei nicht nur als epistemische Kategorie, festgemacht an der Zuverlässigkeit wissenschaftlicher Daten und Methoden, sondern auch als demokratische Kenngröße, gemessen an den Zustimmungsraten zu bestimmten Standpunkten innerhalb der Forschungscommunity. Die Emphase der Einigkeit hat eine neue Metadisziplin hervorgebracht, die Bogner als «empirische Konsensforschung» bezeichnet. ${ }^{13}$ Mit ihr lassen sich zentrale Fragen zum Klimawandel demonstrativ als erledigt abhaken. So hat 2013 eine Forschergruppe aus 12.000 Abstracts von Fachaufsätzen eine Bejahung der menschlichen Ursachen der Erderwärmung errechnet, die sich in den Sphären nordkoreanischer Wahlresultate bewegt. ${ }^{14}$ Die konsensbildende Wirkung der Konsensforschung bleibt jedoch beschränkt. Unter den übriggebliebenen «Dissidenten» traten Gegenexperten hervor, die eigene Auswertungen zum Grad des Konsenses vornahmen und ihn auf eine Minderheitenposition herunter rechneten. ${ }^{15}$ Damit eroberte der Wissenskonflikt ein neues Terrain, wo wahr und falsch in Forschungsfragen per Stimmenzählen geklärt wird. Die Politisierung der Klimawissenschaften beginnt schon auf dem Methodenweg.

Die Konsensfixierung der Klimaforschung hat ihren institutionellen Anker im Weltklimarat (IPCC). Seine Hauptaufgabe besteht darin, den «aktuellen Stand des Wissens über den Klimawandel» in periodisch erscheinenden Berichten festzuhalten. Diese sollen «neutral, politikrelevant, aber nicht politikvorschreibend» sein.

13 Bogner 2021, S. 26-27.

14 Cook et al. 2013.

15 Bogner 2021, S. 28. 
Damit bekennt sich der IPCC zu einer strikten Funktionsteilung zwischen wissenschaftlicher Expertise und politischer Entscheidung. Für die Klimaforscher in den eigenen Reihen ist die Rolle «reiner Wissenschaftler» vorgesehen. Sie legen frei von Interessen Fakten vor, aus denen die Politik Maßnahmen ableitet. Reiner Grundmann und Simone Rödder beschreiben die Rollentrennung mit einer Analogie zur Seefahrt: Experten kartographieren, Politiker navigieren. ${ }^{16}$ Der Vergleich verdeutlicht, warum die Demonstration von Konsens für den IPCC so wichtig ist: Man kann die internationale Politik nicht mit mehreren, voneinander abweichenden Klimakarten auf die Reise schicken. Aufschlussreich ist die Analogie aber vor allem deshalb, weil sie hinkt. Wer die klimatische Zukunft «kartographiert», bildet keine Realitäten, sondern Eventualitäten ab. ${ }^{17}$ Ebenso wenig passt die Analogie, wie Grundmann und Rödder darlegen, zur Praxis der Berichterstattung im Weltklimarat: «Politiker sind an der Kartenerstellung beteiligt». ${ }^{18}$ Die «Summaries for Policymakers», die auf der Basis der IPCC-Berichte entstehen, sind das Resultat intensiver Verhandlungen mit Vertretern der internationalen Politik.

Durch die erfolgreiche Etablierung des IPCC als konsensuales Repräsentationsorgan der Klimawissenschaften konnte sich Dissens mit seinen Positionen fast nur noch in der Form einer fundamentalistischen Alternativexpertise äußern. Weil «Dissidenten», so laut sie auch sein mochten, von den Klimaforschern des IPCC mit strategischer Nichtbeachtung quittiert wurden, entstand ein diskursives Vakuum, das Ende der 2010er Jahre aktivistische Kräfte der Klimabewegung wie Extinction Rebellion und Fridays for Future füllten. Auch sie rechtfertigten sich mit dem wissenschaftlichen Konsens, verbanden mit ihm aber eine höhere politische Verbindlichkeit und Dringlichkeit. Es ging darum, die Wahrnehmung der Erderwärmung durch die internationale Politik vom Modus der schleichenden in jenen der akuten Krise zu versetzen. Dazu brauchte es ungewohnte Protestrituale wie die Mobilisierung von Kindern, die Besetzung von Banken oder die Ausrufung des «Klimanotstands». Sie richteten sich nicht mehr bloß gegen die üblichen Verdächtigen der Anti-Klimapolitik, sondern auch gegen den demokratischen Nationalstaat, dessen Entscheidungsstrukturen als Hindernis für die Krisenbewältigung angesehen wurden. Besonders Aktivisten aus dem Kreis von Extinction Rebellion beklagten ein Versagen der parlamentarischen Demokratie und forderten eine Notstandspolitik auf globaler wie lokaler Ebene, wobei sie für erstere den Weltklimarat und für letztere Bürgerräte mit «moderierenden» Experten vorsahen.

Neu an Extinction Rebellion und Fridays for Future war weniger der Aktivismus an sich, als seine gezielte Ziellosigkeit. Die Klimabewegung verlangte einen «Systemwechsel», verweigerte aber eine Auskunft, was mit ihm zu errei-

16 Grundmann und Rödder 2019, S. 3885.

17 Am ehesten sind Klimamodelle noch mit Weltkarten des frühen 16. Jahrhunderts vergleichbar, als Kartenmacher aus wenigen aufgezeichneten Küstenverläufen die Umrisse ganzer Kontinente ableiteten. Manches war nah an der Wirklichkeit, manches weit davon entfernt, alles ein Produkt der methodisch geschulten Imagination.

18 Grundmann und Rödder 2019, S. 3886.

Leviathan, 49. Jg., Sonderband 38/2021 
chen sei. Der ziellose Aktivismus ist von einem quasi-religiösen Wissenschaftsverständnis beseelt. Will die Menschheit der Selbstausrottung als Strafe für ihre Klimasünden entgehen, muss sie der Wissenschaft folgen. In den Erweckungsparolen «Unite Behind the Science» und «Follow the Science» steckt der Glaube an eine Wissenschaft, in der Epistemisches und Normatives, Sein und Sollen eins wird. ${ }^{19}$ Entsprechend konnte ein Mitglied von Fridays for Future in Deutschland sagen: «Wir formulieren bewusst keine eigenen politischen Ziele, die man dann hinterfragen könnte. Stattdessen stellen wir uns hinter den wissenschaftlichen Konsens. Damit vertreten wir keine persönliche Meinung, sondern eine objektive Empfehlung der Wissenschaft». ${ }^{20}$ Die Auffassung von wissenschaftlichem Konsens erhält hier nochmals eine andere Qualität als in den Verlautbarungen aus der Klimaforschung. Im Einverständnis der Experten steckt schon das politische Programm, und es soll jede weitere Diskussion überflüssig machen.

Dass die Verklärung der Wissenschaft in der Gesellschaft so viel Resonanz erzeugen konnte, hat damit zu tun, dass sie als stärkstes Gegengift wider einen faktenfreien Populismus wahrgenommen wurde. Dadurch aber trieb sie die Polarisierung im Modus des Wissenskonfliktes weiter an. Für eine Thematisierung epistemischer Unsicherheiten und moralischer Dilemmata gab es keinen Platz mehr. Das Wissenschaftsverständnis der Klimabewegung mag noch so unwissenschaftlich sein, es hat für die Klimaforschung Konsequenzen. Erstens wird die Klimaexpertise zum Pars pro Toto für die gesamte Wissenschaft, was selbst im Fall der Erderwärmung zu kurz greift. Der Klimawandel ist eines von vielen Phänomenen der modernen Welt, an dessen Erzeugung, Verschärfung und Bekämpfung die Wissenschaft gleichermaßen beteiligt ist: Ohne sie gäbe es weder Ölplattformen noch Klimamodelle.

Zweitens kollidiert das Verständnis von wissenschaftlicher Expertise als politischer Führerschaft, wie es die Klimabewegung vertritt, mit der Programmatik des Weltklimarates. Wenn sich der IPCC auf die Fahne schreibt, der Politik keine Vorschriften zu machen, läuft der Aufruf, der Wissenschaft zu folgen, ins Leere. Den offiziellen Klimaexperten wird eine Rolle zugeschrieben, die sie gemäß ihrer eigenen Richtlinien nicht einnehmen dürfen. Die falschen Erwartungen, die dadurch geweckt werden, sind aber von Mitgliedern des Weltklimarats kaum je öffentlich thematisiert worden. Vielmehr begrüßten die meisten unter ihnen den Aktivismus aus der Zivilgesellschaft; und einige schlossen sich ihm offen an. Damit aber unterliegen Klimaexperten tatsächlich einem Rollenwandel, wenn auch einem anderen, als es die Klimabewegung suggeriert: Indem sie den Widerspruch zwischen aktivistischer Rollenzuschreibung und eigener Rollenausübung nicht auflösen, erscheinen sie in der Öffentlichkeit als totalisierte Experten, die zugleich gegen die Politik protestieren, die Politik instruieren oder sogar diktieren. Legitimiert wird die Rollenkumulation mit dem Notstand, der ein direktes Eingreifen der Wissenschaft in die Politik gebiete, auch wenn die demokratische Legitimität dafür schwach ist.

19 Siehe dazu Strohschneider 2020, Kap. 7.

20 Maurice Conrad, zitiert in Campillo-Lundbeck 2020. 
Drittens koppelt sich in der Klimafrage die mediale Inszenierung wissenschaftlicher Expertise von ihrer politischen Einbindung ab. Mit der Figur des totalisierten Experten und der Rhetorik des globalen Notstands stellt die Klimabewegung medial auf Krisenmodus um, während die demokratische Politik im Normalzustand bleibt. Viele Regierungen hören Experten zwar an und nehmen die Berichte und Empfehlungen des IPCC ernst, begegnen dem Klimawandel aber mit sukzessiven Anpassungen und langfristigen Reduktionszielen. Der Versuch, eine schleichende Krise durch die Totalisierung der Expertenrolle zu einer akuten Krise zu eskalieren, ist medial gelungen und politisch gescheitert.

\section{Die Eurokrise: Expertokratie auf Wunsch von Exekutiven}

Was einen politischen Normal- von einem Krisenzustand unterscheidet, konnte man vor der Coronakrise schon in der Eurokrise beobachten. Nur war um 2010 die öffentliche Wahrnehmung von Expertise noch viel weniger stark vom Kampf gegen den Klimawandel geprägt als heute. So war auf dem Höhepunkt der Eurokrise das Verhältnis von medialer und politischer Expertise gerade umgekehrt. Die Medien inszenierten einen klassischen Ökonomenstreit, in dem es hauptsächlich um das Für und Wider einer Vergemeinschaftung von Staatsschulden ging, während die Politik eine Krisen-Expertokratie installierte, wie sie europäische Demokratien noch nie gesehen hatten. Mit dem Dringlichkeitsargument, es gelte die Märkte zu beruhigen, um die steigende Zinskurve für Staatsanleihen zu brechen, wurden in Italien und Griechenland gewählte Regierungen zum Rücktritt gedrängt und durch Experten-Kabinette ersetzt. An deren Spitzen standen mit Mario Monti und Loukas Papadimos zwei ehemalige Wirtschaftsprofessoren, die vor ihrer Ernennung hohe Funktionärsposten in der Europäischen Kommission respektive der Europäischen Zentralbank innegehabt hatten. Mit nationalen Expertokratien in Krisenländern war es aber nicht getan. Sie unterstanden, am deutlichsten im Fall Griechenlands, einer supranationalen Expertokratie in der Form der «Troika», bestehend aus Mitgliedern der Europäischen Zentralbank, dem Internationalen Währungsfonds und der Europäischen Kommission. Die Troika sprach die Kreditprogramme, kontrollierte deren Einhaltung und drohte mit Sanktionen.

Ihre Vollmachten entsprangen jedoch keiner expertokratischen Machtergreifung, sondern einer Flucht der demokratischen Politik aus der Verantwortung. Die gewählten Exekutivpolitiker der übrigen EU-Staaten hatten früh erkannt, dass für sie in der Eurokrise nichts zu gewinnen, aber umso mehr zu verlieren war. Die erforderlichen Maßnahmen, von Rettungspaketen bis zu Schuldenerlassen, stießen in der eigenen Bevölkerung auf Ablehnung. Je mehr unpopuläre Aufgaben an die Troika übertragen werden konnten, desto besser fürs eigene Überleben.

Regierungen profitierten in der Eurokrise davon, dass die Wirtschaftswissenschaften im Verlauf des 20. Jahrhunderts eine institutionelle Handlungsfähigkeit entwickelt hatten, wie sie keine andere Forschungsdisziplin besaß. Ihre Expertise beruht auf einer ganzen Reihe von Autoritätsquellen: epistemischen (wie Daten, 
Modelle oder Theorien), instrumentellen (wie Inflationsrate, Konjunkturbarometer und Bruttoinlandprodukt) sowie institutionellen (wie EZB, WTO oder IWF). Anders als in der Klima- oder Gesundheitspolitik genießen Expertenorganisationen in der Wirtschaftspolitik beträchtliche Entscheidungsspielräume, die ihnen im Krisenfall direkte Interventionen ins Marktgeschehen ermöglichen. ${ }^{21}$ Die Politik konnte daher ein expertokratisches Potential aktivieren, das bereits institutionell angelegt war.

Auch den Wirtschaftswissenschaften selbst boten die institutionellen Anker an der Schnittstelle von Politik, Wirtschaft und Wissenschaft nach 2007 einen entscheidenden Halt. Ihre epistemische und instrumentelle Autorität war nach dem Kollaps des globalen Finanzsystems und der staatlichen Bankenrettungen stark geschwächt. Wirtschaftswissenschaftliche Konjunkturmodelle, Markttheorien und Finanzstabilitätsmechanismen hatten in der öffentlichen Wahrnehmung versagt, die Ökonomie ihren Anspruch auf Wissenschaftlichkeit verspielt. Anders als universitär forschende Ökonomen konnten die EZB und der IWF in der Eurokrise expertokratisch durchgreifen, ohne sich für die wissenschaftlichen Grundlagen ihres Vorgehens öffentlich rechtfertigen zu müssen. Die institutionelle Autorität erlaubte es der EZB sogar, zu «unorthodoxen» Mitteln zu greifen, deren Wirksamkeit noch kaum empirisch erwiesen oder theoretisch begründet war. Zwar fanden durchaus Debatten über die krisenpolitische Aufrüstung von Zentralbanken statt, aber am intensivsten dürften sie innerhalb der eigenen institutionellen Mauern geführt worden sein, was den Eindruck einer expertokratischen Selbstregulation nochmals verstärkte. ${ }^{22}$

In der Eurokrise gab es Experten mit exekutiven Vollmachten und Experten mit medialer Breitenwirkung, aber kaum welche, die beide Rollen zugleich ausfüllten. Die Mitglieder der Troika hielten sich in den Medien möglichst bedeckt und kommunizierten öffentlich nur so viel, wie ihnen für die Beruhigung der Märkte erforderlich schien. Die Minister der Krisen-Kabinette wiederum präsentierten sich in der Öffentlichkeit weniger als wissenschaftliche Experten, denn als politische Entscheidungsträger und gaben ihrem Sonderstatus als ungewählte Expertokraten den Schein der demokratischen Normalität. Als sie nach kurzer Krisenintervention auf demokratischem Wege wieder abgewählt wurden, verschwanden sie geräuschlos. Die medial exponierten Ökonomen dagegen machten klare Ansagen, blieben aber, wie ihr Publikum, zu einem Dasein als Zaungäste verdammt. Als solche dominierten sie zumindest den öffentlichen Diskurs über die Krise, zumal sie in den Medien kaum Konkurrenz von Politologen oder Juristen erhielten, die den Blick eher auf die demokratiepolitischen Implikationen der Rettungsmaßnahmen hätten lenken können.

Die Rollentrennung zwischen institutionellen Expertokraten und medialen Experten hatte im Norden Europas ganz andere Folgen als im Süden. In der gesellschaftlichen Wahrnehmung der nördlichen EU-Staaten erschien die politische Bewältigung der Eurokrise weit «normaler», als sie es tatsächlich war. Entscheidend

21 Hirschman und Popp Berman 2021.

22 Baltensperger 2012, S. 9. 
dafür war, dass die Eurokrise für die meisten Menschen nördlich der Alpen und Pyrenäen ein reines Medienereignis blieb, mit Fachleuten aus Forschungsinstituten, die das Geschehen kommentierten. Die Währungskrise machte sich wegen des gefallenen Eurokurses höchstens bei Reisen in Nicht-EU-Länder bemerkbar. Weil die Eindämmung der Krise gelang und die dafür nötigen Maßnahmen nicht auf die Lebensrealität durchschlugen, erschien das krisenpolitische Vorgehen intuitiv unproblematisch. Entsprechend gab es im Norden Europas kaum Widerstand gegen die Einschränkungen der Demokratie innerhalb der Europäischen Union. Die Rechnung der nationalen Exekutiven, sich im Moment der krisenhaften Zuspitzung hinter den Expertokraten von ihren Gnaden zu verstecken, ging auf.

Ganz anders war die Wahrnehmung in den südeuropäischen Ländern, wo die Bevölkerung von den Maßnahmen der Troika hart getroffen wurde. Die von außen auferlegte Austeritätspolitik erschien nicht als ein Akt der Krisenbewältigung, sondern vielmehr der -verschärfung, mit dem Ziel, die EU-Staaten des Nordens vor jedem Schaden zu bewahren. Die Troika wurde als verlängerter Arm der deutschen Bundesregierung wahrgenommen, ausgestattet mit dem Auftrag, deutsche Banken wegen ihrer faulen Kredite in Griechenland schadlos zu halten. Die expertokratische Krisenpolitik traf der Vorwurf einer doppelten Illegitimität: Sie heble die demokratische Selbstbestimmung der südeuropäischen Staaten aus und betreibe nordeuropäische Krisenprävention auf dem Buckel südeuropäischer Opfer der Währungsunion. Die breit geteilte Überzeugung, einem politisch gelenkten Expertendiktat aus dem Ausland zu unterliegen, beflügelte populistische Bewegungen wie Syriza, Cinque Stelle und Podemos, die mit dem Versprechen einer nationalen Basisdemokratie Fundamentalopposition gegen die Austeritätspolitik betrieben. In Ansätzen kam es damit erstmals in einer akuten Krise zur Konfrontation der expertokratischen mit der populistischen Illusion. Syriza brachte mit Yanis Varoufakis einen politischen Ikarus hervor, der in der Doppelrolle des medialen Gegenexperten und populistischen Regierungsministers spektakulär abstürzte. Die Protestbewegungen gewannen Stimmen und teilweise auch Wahlen, vermochten aber ihre Versprechen kaum in Taten umzusetzen.

\section{Coronakrise: nationale Exekutivgewalt und mediale Expertokratie}

Pandemien unterscheiden sich von Finanzkrisen unter anderem dadurch, dass sie sogleich das Leben aller Menschen in einem betroffenen Gebiet tangieren. Die Expertokraten der Eurokrise mussten als erstes die institutionellen Anleger beruhigen; wie sie die breite Bevölkerung erreichten, erschien vorerst nachrangig. Der Ausbruch des Coronavirus stellte die öffentliche Krisenkommunikation vor ganz andere Herausforderungen, und es lag in der Natur der Sache, dass die offizielle Krisenexpertise sogleich eine massenmediale Dimension erhielt, zumal die derzeit lebenden Generationen in Europa und Nordamerika noch nie eine Pandemie erlebt hatten.

Die Gefahr der rasanten Schadensvermehrung hatte die Corona-Pandemie jedoch mit Finanzkrisen gemein: Börsenpanik ist so ansteckend wie das Coronavi-

Leviathan, 49. Jg., Sonderband 38/2021 
rus, und wenn eine unkontrollierte Ausbreitung einsetzt, ist sie nur noch schwer aufzuhalten. Darum kam es in beiden Fällen auf schnelles Eingreifen mit massiver Hebelwirkung an. Eine weitere Gemeinsamkeit der beiden Krisen bestand in ihrer globalen Dimension. Dennoch fiel die politische Reaktion auf die virale Bedrohung ganz anders aus. Sie wurde zum Moment der nationalstaatlichen Exekutiven. Diese waren es, die in fast allen Erdteilen die Initiative an sich rissen. Parlamente brachen laufende Sessionen ab, regionale Regierungen ordneten sich der Zentrale unter, während supranationale Akteure wie die WHO oder die EU von den nationalstaatlichen Maßnahmen überrollt wurden. Regierungen schlossen Grenzen und schränkten die Bewegungs- und Versammlungsfreiheit für das gesamte Staatsgebiet ein.

Die nationale Seuchenpolitik wurde in allen betroffenen Ländern von wissenschaftlicher Expertise begleitet, aber in keinem Staat expertokratisch diktiert. Was aber führte zur Renaissance der nationalstaatlichen Krisenpolitik mit potenzierter Exekutivgewalt? Ein mögliche Antwort wäre: Gesundheitspolitik ist, anders als Finanzpolitik, nach wie vor eine Domäne des Nationalstaats. Das aber erklärt ebenso wenig wie der Zwang zur Krisenkommunikation mit massenmedialer Reichweite, warum die nationalen Exekutiven gerade in der Europäischen Union das Heft so entschieden an sich rissen, ohne sich anfänglich um eine krisenpolitische Koordination untereinander auch nur zu bemühen. Der Hauptgrund dafür waren die Ereignisse in Italien. Genau genommen handelte es sich um zwei verschiedene Vorgänge, wobei einer davon erschreckend war: Es handelte sich um die Bilder aus Bergamo mit überfüllten Korridoren von Kliniken, erschöpftem Pflegepersonal und Kolonnen von Militärwagen, die den Abtransport von Leichen besorgten. Die Bilder verschmolzen zum Worst-Case-Szenario eines Massensterbens in einem überlasteten Gesundheitssystem. Es gab den Regierungen anderer Länder die Legitimationsgrundlage für eine radikale Präventionspolitik in der Form von Geschäfts- und Schulschließungen sowie Einschränkungen der Bewegungs- und Versammlungsfreiheit bis zu Ausgangssperren. Der andere Vorgang dagegen war ermutigend. Er betraf die Beliebtheitswerte der Regierung Conte, die mit jeder Verschärfung der Seuchenbekämpfung weiter nach oben schnellten. Diesen Popularitätseffekt hatte niemand erwartet. Noch wenige Wochen zuvor, als bereits Bilder aus Wuhan mit ähnlichem Aussagehalt wie jene aus Bergamo zirkulierten und eine Ausbreitung des Virus in andere Weltteile wahrscheinlich wurde, schreckte man in Europa vor minimalen Eingriffen zurück, weil man befürchtete, nach den Fehlalarmen der Schweine- und Vogelgrippe mit Präventionsmaßnahmen wie der Unterbindung des interkontinentalen Reiseverkehrs auf Ablehnung zu stoßen. Erst durch die Kombination beider Vorgänge in Italien erschien den nationalen Regierungen in Europa ein energisches Eingreifen opportun. Für eine Schadensabwendung war es zu diesem Zeitpunkt aber schon zu spät. Es konnte nur noch um Schadensminderung gehen.

Wenn die Coronakrise, anders als die Eurokrise, zur Stunde der nationalen Exekutiven wurde, wie konnte dann der Eindruck entstehen, sie habe eine neue Expertokratie der Virologen und Epidemiologen hervorgebracht? Die Diagnose einer Expertenherrschaft fiel nicht nur in Polemiken gegen die Pandemiepolitik, 
sondern auch in Beobachtungen von Juristen und Philosophen, Soziologen und Historikern. ${ }^{23}$ Selbst Ökonomen sprachen in Verkennung der politischen Dimension ihrer eigenen Disziplin von einer «Virokratie», die den Spielraum der Krisenpolitik einschränke. $^{24}$

\subsection{Das mediale Expertenparlament und die Machtakkumulation Drostens}

Der Eindruck einer Machtübernahme durch Experten wurde medial erzeugt. In der Coronakrise erreichte die Epistemisierung des Politischen durch öffentliche Wissenskonflikte unter Experten eine neue Dimension. Entscheidend dafür war nicht unbedingt, dass ihre mediale Präsenz höher war als je zuvor, sondern dass die prominentesten Figuren nach dem Vorbild der Klimakrise die Rolle von totalisierten Experten annahmen. Sie verstanden sich als reine Wissenschaftler, die nur von der «Faktentreue» ihrer Fachkenntnis geleitet sind und sich zu nichts anderem äußern als dem Sachgebiet ihrer Expertise. Damit aber verbanden sie den Anspruch, den pandemiepolitischen Kompass für Regierungen zu stellen. Krisenpolitik wurde zur Erweiterung der fachwissenschaftlichen Kernkompetenz, womit die epistemische Entgrenzung der Expertenrolle einsetzte. Wer Kritik an ihren Wissens- oder Geltungsansprüchen übte, machte sich der «Wissenschaftsleugnung» verdächtig, wer Experten mit anderen politischen Auffassungen anhörte, der «False Balance» schuldig. Die Rolle des aktivistischen Expertokraten im Gewand des reinen Wissenschaftlers erfüllte für ihre Träger die Dreifachfunktion, diskursive Hegemonie zu erreichen, politischen Einfluss auszuüben und Immunität gegen Kritik zu erlangen.

Damit schuf die mediale Inszenierung von Expertise eine alternative Realität des Politischen, die den Blick auf die tatsächlichen Entscheidungsstrukturen verstellte. Zugleich aber bildete sie eine Machtressource von eigener Gesetzmäßigkeit, die Rückwirkungen auf das Regierungshandeln hatte. Eine treibende Kraft in diesem Prozess waren die sozialen Medien, vor allem Twitter, wo sich Nischenforscher im $\mathrm{Nu}$ zu multimedial tätigen Meinungsmachern mit grossem Gefolge mauserten. Twitter wurde zur zentralen Rekrutierungsplattform für Experten, weil sich das Netzwerk schon kurz nach dem Seuchenausbruch in Wuhan als Börse für wissenschaftliche Neuigkeiten zum Coronavirus etabliert hatte. Zu einem Zeitpunkt, als der Erkenntnisstand noch tief, der Handlungsdruck aber bereits gross war, bot sich Twitter wegen seiner eigenen «viralen» Eigenschaften - der Möglichkeit zur rasanten Verbreitung und Verlinkung von Kurznachrichten - als Plattform für Forschende an. Der Vorteil der kommunikativen Geschwindigkeit und globalen Vernetzung überwog den Nachteil der fehlenden Trennung von Spezialisten und Laien. Dadurch entstand eine spezialisierte Massenkommunikation, für die es vor Twitter an technologischen Voraussetzungen gefehlt hatte. Millionen Nutzer konnten mitverfolgen, wie Virologen, Epidemiologen oder Infektiologen neueste Studienergebnisse präsentierten und kommentierten. Was unter Normalbedingun-

23 Nolte 2020; Pollmann 2020; Edenharter 2020; Pfadenhauer 2020; Höffe 2020.

24 Fuster und Eisenring 2020.

Leviathan, 49. Jg., Sonderband 38/2021 
gen in vertraulichen Gutachterverfahren durch Fachleute geschieht, fand nun in aller Öffentlichkeit statt: die Bewertung provisorischer Erkenntnisse und ihre Einbettung in den aktuellen Wissensstand.

Die Pandemie rückte wissenschaftliche Forschungsgebiete ins Zentrum der Aufmerksamkeit, die zuvor kaum beachtet worden waren. Hatten es Journalisten in der Eurokrise bei der Suche nach Experten noch relativ leicht, weil es in den Wirtschaftswissenschaften bekannte Gesichter und einsehbare Forschungsrankings gab, so standen sie nun vor dem Problem, dass sie wenig Kenntnis von den krisenrelevanten Disziplinen und noch weniger von ihren führenden Köpfen hatten. In dieser Situation bot sich die Beobachtung des Kommunikationsverlaufs zu Covid-19 auf Twitter als neue Alternativlösung an, um aus dem globalen Fachdiskurs einheimische Stimmen herauszufiltern, die man in den nationalen Medien zu Corona-Experten aufbauen konnte.

Allerdings konnten sich selbst Wissenschaftsjournalisten aus dem Kommunikationsverhalten von Forschern auf Twitter kaum ein Urteil bilden, wie es um deren fachliche Kompetenz stand. Zu Beginn gaben sie vor allem Männern das Wort, die auf Twitter mit schlagzeilenträchtigen Aussagen zum Virus und seiner politischen Bekämpfung aufgefallen waren. Wie es um ihren Forschungsausweis bestellt war, blieb lange ungeklärt, und auch die Frage, wie sich Spezialkenntnisse über Viruserkrankungen und pandemiepolitischer Sachverstand zueinander verhielten, wurde in den nationalen Medien kaum gestellt. ${ }^{25}$ Insofern legten sie jenen Experten den roten Teppich aus, die ihn als «Stealth Issue Advocates» betreten wollten.

Vom ersten Moment an traten damit Experten als uneingestandene Krisenpolitiker zweiter Ordnung auf. Über Monate schaukelte sich das Geschehen in den sozialen und nationalen Medien wechselseitig hoch. Wer sich auf Twitter das Profil eines urteilsfreudigen Experten gab, erhielt eher eine Einladung ins Fernsehen, wo er mit zugespitzten Aussagen wiederum die Zahl seiner Twitter-Follower in die Höhe treiben konnte, was ihn als Gast für Talkshows noch attraktiver erscheinen ließ. Mit der Politisierung der medialen Krisenexpertise rückten prominente Experten in die Rolle von Repräsentanten für ein bestimmtes Meinungsspektrum. Entsprechend wurden sie in den Medien entweder gegeneinander oder gegen die Regierung in Stellung gebracht. In Deutschland vertrat Christian Drosten den strikten Eindämmungskurs der Bundesregierung, Hendrik Streeck die liberale Lockerungspolitik der CDU-FDP-Koalition in Nordrein-Westfalen und der «alternative» Experte Sucharit Bhakdi die populistische Anti-Pandemiepolitik der AfD. So entstand ein mediales Expertenparlament jenseits demokratischer Institutionen.

Christian Drosten nahm in diesem Parlament von Beginn an eine herausgehobene Stellung ein, weil er über die politische Bühne zu medialer Prominenz gelangte. Den meisten Medienschaffenden wurde er in einer Serie von Pressekonferenzen bekannt. Die erste fand bereits Mitte Februar statt, organisiert von der Nationalen Akademie der Wissenschaften Leopoldina. Drosten saß an der Seite Lothar Wielers, der als Präsident des Robert Koch-Instituts die Sicht des Bundes vertrat.

25 Zum Verhältnis von wissenschaftlicher Reputation und medialer Prominenz siehe Weingart 2001, S. 262-266. 
Als Leiter des vom RKI benannten «Konsiliarlaboratoriums für Coronaviren» an der Charité hatte Drosten eine lose Anbindung an die institutionelle Politikberatung. Ende Februar startete das Coronavirus-Update im Norddeutschen Rundfunk, in dem Drosten täglich für eine rasch wachsende Hörerschaft detaillierte Lageanalysen vornahm, neue Studienergebnisse besprach und politische Eindämmungsschritte erörterte. Anfang März folgten zwei Bundespressekonferenzen des Gesundheitsministeriums, in denen Drosten direkt neben Jens Spahn platziert wurde und viel Redezeit erhielt. Damit war er in der politischen Inszenierung der Regierung der prominentere Experte als Wieler, womit die offizielle Rangordnung der beiden im Feld der Politikberatung auf den Kopf gestellt wurde.

Die zwei Pressekonferenzen reichten Drosten, um fortan Politikberatung per Podcast zu machen. Voraussetzung dafür war der gewaltige Publikumserfolg der Interviews, in denen Drosten das gesellschaftliche Bedürfnis nach Hintergrundinformationen zur viralen Bedrohung mit einer Komplexitätszumutung befriedigte, die in der Wissenschaftsvermittlung neue Standards setzte. So bildete er ein Heer von Laien-Virologen heran, die er, sobald sie sich zur Mitsprache ermächtigt fühlten, als Fachmann wieder auf ihren Platz verweisen musste. Dank der Breitenwirkung der Interviews durfte er damit rechnen, dass seine Aussagen das Ohr der politisch Verantwortlichen fanden. ${ }^{26}$ Im NDR-Podcast verband sich öffentliche Aufklärung mit pandemiepolitischer Intervention, am auffälligsten in der bekannten Episode Ende April 2020, als Drosten Daten aus dem eigenen Labor zur Viruslast im Rachen von Kindern statistisch aufbereiten ließ, die provisorischen Ergebnisse als Preprint veröffentlichte und sie, obwohl ihre Validierung durch die Fachgemeinschaft noch ausstand, sogleich für eine massenmediale Warnung vor einer «unbegrenzten Öffnung» von Schulen und Kindergarten einsetzte. Dieses Vorgehen entsprach bereits dem Modell der «Stealth Issue Advocacy», weil Drosten die Infektiosität in der Frage der Schulöffnungen zum Leitkriterium erhob, ohne sich und sein Publikum darüber aufzuklären, dass er eine normative und keine wissenschaftliche Setzung vornahm, weshalb er auch nicht als «Wissenschaftler» sprach, sondern als Aktivist, der alles der Pandemiekontrolle unterordnete und daher Werte wie Bildungs- und Generationengerechtigkeit schwächer gewichtete als die - erst ansatzweise geklärte - Ansteckungsgefahr durch Kinder. Die versteckte Normativität seiner Position kam in den Medien kaum zur Sprache, schon gar nicht, als sich die Bild-Zeitung anmaßte, einen Wissenskonflikt mit Drosten anzuzetteln, indem sie ihm, ausgerüstet mit einer Blütenlese kritischer Kommentare von Fachspezialisten zur Kinderstudie, wissenschaftliches Versagen vorwarf. Die Anfeindungen aus dem Boulevard und ihre erfolgreiche Abwehr per Twitter bestärkten Drosten in seiner Rolle als versteckten Aktivisten. Je polarisierter das pandemiepolitische Klima wurde, desto weniger konnten die Komplikationen, die mit der Rolle verbunden sind, adressiert werden.

26 Die Distanz zur Bundespressekonferenz hatte für ihn den zusätzlichen Vorteil, dass er sich nicht mehr den politischen Journalisten mit ihrem konfrontativen Habitus stellen musste, sondern auf die kompetentere und affirmativere Befragung durch Wissenschaftsjournalisten zählen konnte.

Leviathan, 49. Jg., Sonderband 38/2021 
Tatsächlich wurde Drostens mediale Macht durch Twitter potenziert. 2020 verzeichnete er auf der Plattform das grösste Wachstum aller deutschen Nutzer. Die Anzahl seiner Follower stieg im Verlauf der Pandemie um mehr als das Tausendfache auf eine Dreiviertelmillion. Aus Drosten wurde ein massenmedialer Einmannbetrieb, der mit ein paar Tweets die Bild-Zeitung in die Schranken weisen und konkurrierende Experten in den Senkel stellen konnte. Setzte er einen Tweet $\mathrm{ab}$, verbreiteten ihn Zeitungen, Fernsehen und Radio weiter, meist mit affirmativem Unterton. So viel mediale Macht hat noch nie ein einzelner Forscher auf sich vereint.

\subsection{Eine aktivistische Experteninstitution im Dienste der Regierung}

Mit der Zuspitzung der pandemischen Lage im Herbst 2020 kam es wieder vermehrt zu gemeinsamen Auftritten von Wieler und Drosten, zuerst im bekannten Rahmen der Bundespressekonferenz und dann, als der Wissenskonflikt zwischen Befürwortern und Gegnern eines zweiten Lockdown auf eine Polarisierungsblockade zutrieb, in einer gänzlich neuen Konstellation, die für den weiteren Pandemieverlauf wegweisend war. Es handelte sich um einen Akt der «Stealth Issue Advocacy» mit staatlicher Unterstützung. Am 8. Dezember 2020veröffentlichte die Leopoldina eine Stellungnahme, die einen harten Lockdown «aus wissenschaftlicher Sicht» für «unbedingt notwendig» erklärte. ${ }^{27}$ In Stil und Aufbau hatte sie den Charakter eines Appells der Wissenschaft an die Politik. Verglichen mit ihren früheren Stellungnahmen zur Pandemiepolitik, die bereits reich an Imperativen waren, verschärfte die Leopoldina den Verbindlichkeitsduktus ihrer Sprache nochmals. Das Resultat war eine widersprüchliche Rhetorik, die ein Vorgehen «vorschlug», dessen einzelne Schritte anschließend in «muss»-Form ausformuliert wurden. Zur Untermauerung der Imperative dienten wenige Illustrationen, deren Daten eine deterministische Deutung erhielten. Mit ihnen verallgemeinerte die Stellungnahme ein einzelnes Fallbeispiel, Irland, zum visualisierten Gesamtbefund, «strenge Maßnahmen über einen kurzen Zeitraum» würden die Fallzahlen deutlich senken. Die Stellungnahme schloss mit einer Liste von Unterzeichnern, von denen viele den Status prominenter Experten hatten.

Der bekannteste Name auf dem Leopoldina-Manifest war jener von Drosten. Kaum war die Stellungnahme veröffentlicht, erklärte er sie in einem nächsten rhetorischen Eskalationsschritt zur «letzten Warnung der Wissenschaft an die Politik». Werde die Warnung ignoriert, habe sich die Politik «nicht mehr für die Wissenschaft entschieden». ${ }^{28}$ Seine Worte hallten in den Medien nach und schienen in der Politik Wirkung zu zeigen. Kanzlerin Merkel trat tags darauf als Vollstreckerin des wissenschaftlichen Willens auf, als sie im Bundestag die Notwendigkeit eines harten Lockdowns in einer emotionalen, an ihre Studienwahl der Physik erinnernden Rede mit dem Argument begründete, man könne vieles außer Kraft setzen, aber «die Schwerkraft nicht, die Lichtgeschwindigkeit nicht -

27 Leopoldina 2020, S. 1.

28 Rehrmann 2020. 
und andere Fakten auch nicht». Der Appell der Leopoldina hatte sich vor dem Parlament in einen wissenschaftlichen Sachzwang verwandelt. Die «Zahlen», wie es die Kanzlerin sagte, «sind, wie sie sind», und sie erforderten den Lockdown. ${ }^{29}$

Die Ereignisse, die der Verhängung des zweiten Lockdowns vorausgingen, sehen auf den ersten Blick wie ein Lehrstück in wissenschaftlicher Durchsetzungskraft gegenüber der Politik aus. So wurden sie in den nationalen Medien auch dargestellt. Der Eindruck aber täuscht. Hier stand nicht eine agierende Wissenschaft einer reagierenden Politik gegenüber, und schon gar nicht stellten Experten Entscheidungsträger ultimativ vor die Wahl, entweder der Wissenschaft zu folgen oder ohne sie ins Verderben zu stürzen. Vielmehr saß die Politik bei der Abfassung der Warnung an sich selbst von Anbeginn mit am Tisch, personifiziert durch Lothar Wieler, der das Papier in seiner Funktion als Präsident des Robert Koch-Instituts unterzeichnete. Wieler ist als Leiter einer Bundesbehörde dem Gesundheitsminister weisungsunterworfen. Äußert er sich öffentlich, tut er dies als Vertreter des Staates. Er hat gegenüber der Öffentlichkeit keine Stimme, die von der Stimme der Bundesregierung getrennt werden könnte. Da es keine Anhaltspunkte gibt, dass Wieler mit der Mitwirkung an der Stellungnahme seinen Dienstherrn hintergangen hat, um ihn durch eine eklatante Kompetenzüberschreitung öffentlich unter Druck zu setzen, ist nur ein Schluss möglich: Durch den Präsidenten des RKI signierte die Bundesregierung, die sich zu diesem Zeitpunkt bereits für die Verhängung eines zweiten Lockdown entschieden haben musste, die Stellungnahme mit. Die Leopoldina ihrerseits gab mit der Unterschrift ihre eigene Unabhängigkeit von der Politik preis. ${ }^{30}$

Hätten die Experten der Leopoldina die Rolle ehrlicher Vermittler gewählt, um der Politik in einer besonders akuten Krisenphase die Chancen und Risiken verschiedener Handlungsoptionen auf wissenschaftlicher Grundlage zu erläutern, wäre die fehlende Unabhängigkeit Wielers kaum ins Gewicht gefallen. Problematisch wurde sie erst durch ihren Auftritt als versteckte Aktivisten. Fordert ein weisungsgebundener Verwaltungsbeamter die eigene Regierung im Namen der Wissenschaft öffentlich zu einem richtungsweisenden Krisenentscheid auf, stimmt etwas grundlegend nicht.

Warum aber kam die Nationale Akademie der Wissenschaften überhaupt auf die Idee, Wieler für die Stellungnahme einzubinden? Der Schritt ist nur nachvollziehbar, wenn man die Leopoldina-Expertise auf die Funktion reduziert, die Legitimation für den Lockdown-Entscheid der Bundesregierung zu maximieren. Wieler war nach Drosten der medial prominenteste Pandemieexperte; seine abhängige Stellung wurde von den Medien ausgeblendet. Die Stimme der versammelten Experten sollte dem Regierungsbeschluss so viel öffentliche Zustimmung sichern, dass sich eine demokratische Diskussion seines Für und Wider erübrigte. Was

29 Merkel 2020.

30 Dass daneben auch mehrere Dutzend prominente Forschende, die nicht den spezifischen Einschränkungen Wielers unterliegen, ihren Namen unter die Stellungnahme gesetzt haben, vermochte die Verstrickung nicht aufzulösen. Die Voraussetzung einer unabhängigen Politikberatung war nicht gegeben.

Leviathan, 49. Jg., Sonderband 38/2021 
als Warnung der Wissenschaft an die Politik ausgegeben wurde, war in Wirklichkeit eine Drohung der Bundesregierung an den Bundestag und die Ministerpräsidentenkonferenz. Den Parlamentariern und Landeshäuptern drohte die Ächtung durch die Nationale Akademie der Wissenschaften, sollten sie die Notwendigkeit eines harten Lockdowns weiter debattieren oder gar in Frage stellen wollen. Das Kalkül ging auf. Nur die Rechtspopulisten protestierten im Bundestag gegen die Verschärfung der Einschränkungen und beflügelten die Bundeskanzlerin zu ihrem Plädoyer für die Wissenschaft, das von den meisten Medien begeistert aufgenommen wurde.

\subsection{Der Preis der legitimatorischen Instrumentalisierung von Expertise}

Wo liegt das Problem, wenn eine Regierung die Empfehlung ihrer Experten ernst nimmt und deren Renommee anschließend für eine Kampagne zur Durchsetzung der Empfehlung einsetzt? Da ist zunächst auf der kommunikativen Ebene die Unaufrichtigkeit, mit der die Bundesregierung und die Leopoldina das gemeinsame Vorgehen als Imperativ der Wissenschaft an die Politik ausgaben. Die Kanzlerin stellte sich vor dem Bundestag als Vollzugsinstanz eines wissenschaftlichen Willens dar («Wenn die Wissenschaft uns geradezu anfleht»), der sich in Wirklichkeit nur dank ihrer Unterstützung so artikulieren konnte. Aus legitimatorischen Gründen evozierte die Politik den Eindruck eines expertokratischen Durchgreifens, ohne dabei ihre Entscheidungsgewalt aus der Hand zu geben. Strategisch eingesetzte Unaufrichtigkeit gehört zum politischen Geschäft, aber hier spielt sie Rechtspopulisten, die in Experten nur zu gern den verlängerten Arm des Establishments sehen, direkt in die Hände.

Ein zweites Problem besteht darin, dass die Leopoldina und die Kanzlerin die Frage des Pro oder Kontra eines harten Lockdowns so gerahmt haben, als lasse sie sich wissenschaftlich beantworten. Die Zahlen mögen sein, wie sie wollen, sie geben in einer komplexen Krise nie vor, was zu tun ist. Die Leopoldina ihrerseits inszenierte einen wissenschaftlichen Konsens, wo es keinen geben kann. Ihre Stellungnahme war das Produkt einer Vereinigung von normativ Gleichgestimmten, die Pandemiepolitik machen wollten, als handle es sich um "Tornado Politics». ${ }^{31}$ Der Entscheid, für den Gesundheitsschutz die Freiheitsrechte noch stärker einzuschränken, kann nur der Überzeugung, nicht aber der Erkenntnis entspringen, dass der dadurch verhinderte Schaden den neu verursachten Schaden überwiegt. Es geht primär um Werte und nicht um Wissen, weil materielle und immaterielle Güter - vom Schutz des Lebens über das Verhindern von Elend und das Recht auf Bildung bis zur Wahrung der Generationengerechtigkeit - gegeneinander abgewogen werden müssen. Gleichzeitig kann die Überzeugung nur provisorischen Charakter haben, denn die Werteprioritäten sind an Annahmen gebunden, die durch den Pandemieverlauf laufend widerlegt werden können. Um die Überzeugung in einem demokratischen Entscheidungsprozess durchzusetzen, bedarf sie

31 Pielke 2007, S. 43. Zur Vermischung von Wissen und Werten in der wissenschaftlichen Politikberatung siehe Weingart 2001, S. 149. 
einer mehrheitsfähigen Begründung. Das bedeutet aber, dass sich jene, die sie vorbringen, einer Debatte stellen müssen, in Anerkennung der Tatsache, dass es auch mitten in einer Krise legitime Gründe für andere Werteprioritäten gibt. ${ }^{32}$ Genau das wollte die Bundesregierung vor dem zweiten Lockdown mit Unterstützung der Leopoldina verhindern - nicht weil sie keine guten Argumente für einen Lockdown gehabt hätte, sondern weil sie alle Akteure, die von ihrer Wertepriorisierung abwichen, ins Abseits stellen wollte. Die wissenschaftliche Wahrheit, repräsentiert durch die Leopoldina, wurde wie ein Wert behandelt, der alle anderen Werte aus dem Feld schlug. Die Wissenschaft verwandelte sich in Ideologie.

Die expertokratische Illusion, die hier in ihrer ganzen Polarisierungskraft zum Tragen kam, wirkte sich besonders drastisch auf Experten aus, die sich zuvor in den Medien gegen einen Lockdown ausgesprochen hatten. Sie standen nun nicht als Unterlegene in einer politischen Auseinandersetzung, sondern als unqualifizierte und damit gefährliche Forscher da. Sieg und Niederlage in einer politischen Kontroverse schlugen direkt auf die An- und Aberkennung von wissenschaftlicher Reputation durch. Journalistinnen des Spiegel stellten in einem Interview mit Drosten die Behauptung in den Raum: «Einen größeren Schaden als CoronaLeugner haben im vergangenen Jahr wohl Experten angerichtet, die immer wieder gegen wissenschaftlich begründete Maßnahmen argumentiert haben, zum Beispiel Jonas Schmidt-Chanasit und Hendrik Streeck».33 Drosten widersprach nicht, sondern rückte Streeck, immerhin sein eigener Nachfolger auf dem Lehrstuhl für Virologie an der Universität Bonn, im NDR seinerseits in die Nähe der «Wissenschaftsleugnung» - weil er gesagt hatte, man müsse «mit dem Virus leben lernen». ${ }^{34} \mathrm{Im}$ politischen Triumph über seine Widersacher aus dem Pandemieherbst, den ihm die Bundesregierung mit der «Befolgung» der Leopoldina-Stellungnahme beschert hatte, erhob Drosten einen expertokratischen Souveränitätsanspruch, mit dem er jeden pandemiepolitischen Dissens als wissenschaftsfeindlich abtun und Figuren wie Streeck zu halben Bhakdis degradieren konnte. Spätestens im Frühjahr 2021 war Drosten zur Personifikation des totalisierten Experten geworden.

Ein drittes Problem des gemeinsamen Vorgehens von Bundesregierung und Leopoldina liegt in der Entmündigung des Parlaments. Gibt eine Regierung einen schwerwiegenden Entscheid als wissenschaftlichen Imperativ aus, zwingt sie alle, die nicht als Feinde der Wissenschaft dastehen wollen, zu blinder Gefolgschaft und lässt politischen Widerspruch nur noch in der Form einer populistischen Fundamentalopposition zu. Man sieht hier, wie stark sich expertokratische und populistische Illusionen wechselseitig bedingen. Die Entmündigung geht aber noch weiter. Die Stellungnahme vermittelte den Parlamentsmitgliedern mit dem alleinigen Bezug auf Irland die trügerische Gewissheit, wenige Wochen - die Zeit der «Weihnachtspause» - reichten aus, um die Ansteckungszahlen markant zu drücken. Sie beschränkte sich auf ein Best-Case-Szenario, ohne dieses als solches

32 Siehe dazu auch Deutscher Ethikrat 2020, S. 2-3.

33 Bredow und Hackenbroch 2021.

34 Siehe dazu Benner 2021.

Leviathan, 49. Jg., Sonderband 38/2021 
kenntlich zu machen. Dass die Zahlen nach der Aufhebung des Lockdowns gleich wieder nach oben schnellen könnten, wurde ebenso wenig angesprochen wie die Möglichkeit, dass sie nur langsam sinken könnten. Die Stellungnahme blendete damit die Szenarien, die sich in Irland und Deutschland nach Weihnachten real abspielen sollten, aus. Sie verunmöglichte gerade, was eine output-orientierte Expertise eigentlich gewährleisten müsste: eine möglichst sachgerechte Lageeinschätzung, damit sich Entscheidungsträger für alle Eventualitäten wappnen können.

Der Expertenaufruf der Leopoldina vom 8. Dezember 2020 entfachte ein legitimatorisches Strohfeuer für die Bundesregierung und hinterließ verbrannte Erde für die langfristige Glaubwürdigkeit von wissenschaftlicher Politikberatung. Der kurze «Lockdown in der Weihnachtspause» verfehlte die versprochene Wirkung und mündete in einen monatelangen Stillstand des gesellschaftlichen Lebens bei hohen Todeszahlen. Die Experten, die sich im Herbst gegen drastische Verschärfungen ausgesprochen hatten, waren politisch zu stark geschwächt, um die Bundesregierung zum Überdenken der harten Maßnahmen anzuregen. Stattdessen gingen Mitunterzeichnerinnen der Leopoldina-Stellungnahme mit der Forderung in die Medien, die Schraube so lange weiter anzuziehen, bis die Ansteckungen gegen Null tendierten. Mit welchen Zwangsmaßnahmen diese «No-Covid-Strategie» im Moment der beginnenden Impfkampagne durchzusetzen sei, blieb offen. Ihre Plausibilität hing davon ab, ob epidemiologische Prognosemodelle bei jedem Öffnungsschritt einen rasanten Neuanstieg der Infektionszahlen anzeigten. Das taten sie bis weit in den Frühling 2021 hinein, und so verhängte die deutsche Politik noch Ende April nächtliche Ausgangssperren. Zu diesem Zeitpunkt aber hatten die Fallzahlen schon zu sinken begonnen. Konfrontiert mit der unerwarteten Wende, rang die mediale Expertenprominenz um Drosten um Erklärungen. Man sprach vom «Präventionsparadox», meinte damit eine «Self-Defeating Prophecy» und unterstellte, schwarze Fehlprognosen seien eine effektive Präventionsmaßnahme. Der expertokratische Souveränitätsanspruch fiel in sich zusammen, und Freiheitseinschränkungen, die mit ihm durchgesetzt wurden, verloren ihre Berechtigung. Wer durch die Medien Politikberatung betreibt, wird zum Gefangenen der eigenen Rolle. Früher oder später verwandelt sich das legitimatorische Gewicht der persönlichen Prominenz in eine krisenpolitische Last.

\section{Fazit}

Die Corona-Pandemie hat ein Paradox auf die Spitze getrieben, das der Organisation von wissenschaftlicher Expertise seit jeher innewohnt: die Ausübung der Expertenrolle gilt als Ausdruck höchster Professionalität, aber es gibt keine professionelle Ausbildung zum Experten. Expertise kann man nicht lernen, man wächst in die Rolle hinein. Das Paradox betrifft sowohl die Expertentätigkeit in den Medien als auch jene in der Politik. Beide Systeme inszenieren Expertise, als sei sie die natürliche Fortsetzung der spezialisierten Forschungskommunikation, und verschleiern damit ihren fundamental anderen Charakter, bedingt durch die doppelte Asymmetrie zwischen Spezialistin und Laie beziehungsweise zwischen Sach- 
kompetenz und Entscheidungsgewalt. Historisch ist das Paradox darin begründet, dass sich die Expertenrolle in einem institutionellen Gefüge herausgebildet hat, wo sie in formelle Verfahren der Wahrheits-, Urteils- und Entscheidungsfindung eingebettet war. Das war zuerst das Gericht und seit dem 18. Jahrhundert auch die Politik. ${ }^{35}$ Wurde ein Experte als Sachverständiger in einem Gerichtsverfahren angehört, war seine Rolle durch das Gesetz so präzise festgeschrieben, dass er nur schwer aus ihr herausfallen konnte. Das institutionelle Korsett sorgte dafür, dass die Expertentätigkeit nicht durch eine formelle Ausbildung inkorporiert werden musste.

Heutige Experten-Interviews haben noch immer die Grundstruktur der Befragung von Sachverständigen im Gericht. Ansonsten aber ist die Rolle aus dem institutionellen Rahmen gefallen. Experten können als Sachverständige, Ankläger und Richter auftreten oder ein Verfahren öffentlich kritisieren, an dem sie formell beteiligt sind. Die ihnen attestierte Kompetenz, einen kleinen Ausschnitt der Realität besonders scharf zu sehen, weitet sich in Krisensituationen zum Anspruch aus, der ganzen Gesellschaft den Weg zu weisen. Die Expertenrolle erfährt eine epistemische Entgrenzung und normative Totalisierung. Das Fehlen einer professionellen Schulung ermöglicht einen Wildwuchs an unvereinbaren Rollenentwürfen, die einzelnen Forschern mehr Aufmerksamkeit und Deutungsmacht verleihen, zugleich aber zu Kompetenzüberschreitungen führen und die Konfliktträchtigkeit ihres Handelns verschärfen. Expertise vermischt sich mit Engagement, die Figur des Sachverständigen mit jener des Aktivisten und des Intellektuellen. Krisen werden zum Hebel, um aus der Rollenkumulation politische Macht zu schlagen.

Die Faktoren, die zur Totalisierung der Expertenrolle geführt haben, sind vielfältig: Das Wiedererstarken einer positivistischen Wissenschaftsemphase im Kampf gegen Populismus und Desinformation gehört ebenso dazu wie die Möglichkeit zur digitalen Selbstzeugung von Experten auf Twitter und die Instrumentalisierung der Wissenschaft durch demokratische Regierungen zur Auslagerung unpopulärer Entscheide oder Unterdrückung kontroverser Debatten. All diese Faktoren werden in akuten Krisen, wo Sofort-Expertise für das Verständnis und die Kontrolle der Vorgänge unerlässlich ist, potenziert.

Die Totalisierung der Expertenrolle hat eine ideologische Grundlage, die von der Strahlkraft der Wissenschaft zehrt, aber ihre Möglichkeiten überschreitet. Es ist das Dogma des Sachzwangs, wonach in wissenschaftlichen Befunden der Schlüssel zum politischen Handeln liege. Der Weg vom Sein zum Sollen wird als kurz, gerade und zwingend imaginiert. Krisen bieten sich für die Beschwörung von Sachzwängen deshalb an, weil ihre Bekämpfung klare Ziele und schnelles Handeln erfordert. Um den Klimawandel zu bremsen, muss der Ausstoss von Treibhausgasen reduziert werden, und um eine Pandemie zu kontrollieren, muss das exponentielle Wachstum der Ansteckungen gebrochen werden. Welche Maßnahmen in welchem Umfang zur Erreichung der Ziele notwendig, erlaubt und akzeptabel sind, bleibt jedoch eine politische Frage, die gerade in komplexen Krisen mit hoher Unsicherheit behaftet ist und selbst bei bester wissenschaftlicher

35 Hirschi 2018, S. 59-62.

Leviathan, 49. Jg., Sonderband 38/2021 
Erkenntnisgrundlage divergierende Antworten erlaubt. Unsicherheit gibt es nicht ohne Entscheidungsfreiheit.

Wer Expertise mit der Behauptung von Sachzwängen verknüpft, setzt die Werteprioritäten bestimmter Forschender absolut und verschleiert sie zugleich. Jedes Sachzwangargument ist wertebasiert, denn es bezieht sich auf ein gesellschaftliches Wohl wie Gesundheit, Prosperität oder Nachhaltigkeit, das nur auf einem Weg gesichert werden könne. Entsprechend wäre die Expertokratie eine politische Ordnung, die nur unter der Voraussetzung eines normativen Fundamentalkonsenses in der Gesellschaft existieren könnte, so dass ihre Werteprioritäten hinter dem Schleier wissenschaftlicher Fakten verschwinden würden. Wissenschaft gerinnt, wenn von Sachzwängen die Rede ist, zwangsläufig zur Ideologie, das heisst zu einem Glaubenssystem, das nicht mehr in der Lage ist, zwischen Wissen und Werten zu unterscheiden. Darin liegt die Hauptgefahr der expertokratischen Illusion für die Demokratie, deren pluralistische Ordnung darauf angewiesen ist, dass Werteprioritäten als solche erkennbar sind und Wertekonflikte in öffentlichen Diskussionen ausgetragen werden. Gelingt das nicht mehr, stehen sich in der politischen Auseinandersetzung irgendwann nur noch zwei polare Antipluralismen gegenüber: der expertokratische und der populistische.

Was wäre zu tun, um der Totalisierung der Expertenrolle entgegenzuwirken? Zunächst würde es darum gehen, jene Funktion von Expertise zu stärken, die nur durch sie erfüllt werden kann. Das ist die Erläuterung und Einordnung von aktueller Evidenz für Laien, damit diese komplexe Probleme besser verstehen und mit ihnen kompetenter umgehen können. Expertise ist für diejenigen, denen sie zugute kommt, im besten Fall eine Schule zur Mündigkeit in einem bestimmten, eng begrenzten Handlungsfeld. Damit das gelingt, müssen Experten Handlungsmöglichkeiten eröffnen, nicht Handlungszwänge erzeugen. Das geht nur, wenn Expertise so organisiert wird, dass sie auf der Basis der dargelegten Evidenz verschiedene Entscheidungsszenarien mit ihren jeweiligen Chancen und Risiken, aber auch ihren Wertedilemmata aufzeigt, und es gezielt unterlässt, sich für ein bestimmtes Szenario auszusprechen. Die ideale Rolle dafür ist Pielkes «Honest Broker of Policy Alternatives». Die Norm der «Neutralität» von Experten erhält dadurch erst ihre volle Bedeutung. Sie hebt Experten nicht nur von Lobbyisten, sondern auch von Aktivisten ab. Neutralität bedeutet dabei nicht Wertefreiheit, im Gegenteil, sie bedingt die gezielte Sichtbarmachung der normativen Gesichtspunkte jeder wissenschaftlichen Position. Den Beratenen wird die Freiheit, aber auch die Verantwortung übertragen, sich aufgrund der Expertise selbst ein Urteil zu bilden und die aus ihrer Sicht bestmögliche Entscheidung zu treffen. Eine dezidiert ergebnisoffene Organisation von Expertise ist auch das beste Gegengift gegen ihre Politisierung. Regierungen können Maßnahmen nicht mehr mit dem Argument rechtfertigen, sie würden der Stimme der Wissenschaft folgen, Experten können ihre Evidenz nicht mehr in einer Einseitigkeit präsentieren, dass sie zum Trugschluss einer einzig wissenschaftlich vertretbaren Entscheidung verleitet, und Medien können Experten nicht mehr so leicht gegeneinander in Stellung bringen, damit sie untereinander politische Kontroversen austragen. 
Die Stärkung der Neutralität von Expertise bedingt auch eine konsequentere Trennung der Rollen, in denen Forschende politischen Einfluss ausüben. Wer als Experte Politik berät, kann nicht gleichzeitig als Aktivist auf Twitter auftreten, als Kritiker in den Medien Stellung beziehen oder als Intellektueller Manifeste unterschreiben. Rollen können getauscht, aber nicht kumuliert werden. Die Entflechtung würde nicht nur der Totalisierung der Expertenrolle entgegenwirken, sondern komplementäre Rollen von Forschenden mit ihren spezifischen Eigenschaften aufwerten. So bedingt der Einsatz für ein allgemeines Anliegen eine persönliche Exponierung, der Kampf gegen Desinformation ein öffentliches Engagement und die Kritik an der Politik eine distanzierte Unabhängigkeit, die mit den Bedingungen wissenschaftlicher Politikberatung nicht vereinbar sind. Wissenschaft kann politisch einflussreicher sein, wenn sie das Potential komplementärer Rollen ausschöpft.

Damit die Rollentrennung gelingen kann, muss zuletzt die Tätigkeit von Experten wieder durch klare Regeln und Normen institutionell eingerahmt werden. Politik und Medien können das nicht in Eigenregie schaffen, solange beide, wenn auch in teilweise antagonistischer Aufstellung, aus der Totalisierung der Expertenrolle Profit schlagen. Es braucht dafür qualifizierten Druck von aussen, vor allem aus der Wissenschaftsforschung selbst.

Ein Anfang wäre gemacht, wenn sich unter Experten, Journalisten und Politikern die Einsicht durchsetzen würde, dass eine Demokratie dann am stärksten ist, wenn politische Entscheidungen so viel wie möglich auf wissenschaftliche Expertise gestützt sind und so wenig wie möglich mit ihr gerechtfertigt werden. Die Einsicht würde der Selbstbescheidung der Wissenschaft Rechnung tragen, an die Sheila Jasanoff 2017 unter dem Eindruck von Donald Trumps Wahl erinnert hat: «Science and democracy, at their best, are modest enterprises because both are mistrustful of their own authority». ${ }^{36}$

\section{Literatur}

Baltensperger, Ernst 2012. "Assessing the European Central Bank's Euro Crisis Policies», in CESifo DICE Report 1, S. 9-13.

Beck, Silke 2010. "Moving Beyond the Linear Model of Expertise? IPCC and the Test of Adaptation", in Regional Environmental Change 11, S. 297-306.

Benner, Thorsten 2021. "Die gewünschte Schockwirkung erzielen», in Cicero, 05.05.2021, https://www.cicero.de/innenpolitik/wissenschaft-politik-corona-drosten-kerber-spahn (Zugriff vom 01.07.2021).

Bogner, Alexander 2021. Die Epistemisierung des Politischen. Wie die Macht des Wissens die Demokratie gefährdet. Stuttgart: Reclam.

Brennan, Jason 2016. Against Democracy. Princeton: Princeton University Press.

Campillo-Lundbeck, Santiago 2020. Was Werber von Fridays For Future über gutes Kampagnenmanagement lernen können. https://www.horizont.net/marketing/nachrichten/maurice -conrad-was-werber-von-fridays-for-future-ueber-gutes-kampagnenmanagement-lernen-ko ennen-180489 (Zugriff vom 15.01.2021).

36 Jasanoff 2017, S. 28.

Leviathan, 49. Jg., Sonderband 38/2021 
Cook, John; Nuccitelli, Dana; Green, Sarah A.; Richardson, Mark; Winker, Bärbel; Painting, Rob; Way, Robert; Jacobs, Peter; Skuce, Andrew 2013. Quantifying the Consensus on Anthropogenic Global Warming in the Scientific Literature.https://iopscience.iop.org/articl e/10.1088/1748-9326/8/2/024024/pdf (Zugriff vom 01.07.2021).

Deutscher Ethikrat 2020. Solidarität und Verantwortung in der Corona-Krise. Ad-hoc-Empfehlung. Berlin, 27.03.2020.

Edenharter, Andrea 2020. «Es darf in der Coronakrise keine Denk- und Redeverbote geben», Interview in Deutschlandfunk Kultur, 11.04.2020.

Eyal, Gil 2019. The Crisis of Expertise. Cambridge UK: Polity.

Fuster, Thomas; Eisenring, Christoph 2020. «Corona-Krise: So wollen Schweizer Ökonomen das Land vom Lockdown befreien", in Neue Zürcher Zeitung, 11.04.2020.

Grundmann, Reiner; Rödder, Simone 2019. «Sociological Perspectives on Earth System Modeling», in Journal of Advances in Modeling Earth Systems 11, S. 3878-3892.

Bredow, Rafaela von; Hackenbroch, Veronika 2021. Ich habe schlimme Befürchtungen, was sonst im Frühjahr oder Sommer passieren könnte. https://www.spiegel.de/wissenschaft/me dizin/christian-drosten-wir-muessen-durchhalten-und-vor-allem-auf-die-bremse-treten-a-92 68683b-0415-4f09-b9f5-773bf2215cc1 (Zugriff vom 01.07.2021).

Hirschi, Caspar 2018. Skandalexperten. Expertenskandale. Zur Geschichte eines Gegenwartsproblems. Berlin: Matthes \& Seitz.

Hirschi, Caspar 2021a. «Kalkül schlägt Kompetenz», in Frankfurter Allgemeine Zeitung, 09.03.2021, S. 9-11.

Hirschi, Caspar 2021b. "Weder wissenschaftlich noch demokratisch legitimiert», in Frankfurter Allgemeine Zeitung, 19.03.2021, S. 11.

Hirschman, Daniel; Popp Berman, Elizabeth 2021. «Do Economists Make Policies? On the Political Effects of Economics», in Socio-Economic Review 12, 4, S. 779-811.

Höffe, Ottfried 2020. «Es gibt ein Recht, keine unnötigen Ängste haben zu müssen: ein paar Fragen zum Leben in der Virokratie», in Neue Zürcher Zeitung, 16.12.2020.

Jasanoff, Sheila 2003. "(No?)Accounting for expertise», in Science and Public Policy 30, 3 , S. $157-162$.

Jasanoff, Sheila 2017. «Back from the Brink: Truth and Trust in the Public Sphere», in Issues in Science and Technology 33, 4, S. 25-28.

Leopoldina 2020. Coronavirus-Pandemie: Die Feiertage und den Jahreswechsel für einen harten Lockdown nutzen. 7. Ad-hoc-Stellungnahme. Berlin, Halle, 08.12.2020.

Merkel, Angela 2020. Rede zum Haushaltsgesetz 2021 vor dem Deutschen Bundestag am 9. Dezember 2020 in Berlin. https://www.bundesregierung.de/breg-de/service/bulletin/rede -von-bundeskanzlerin-dr-angela-merkel-1826624 (Zugriff vom 04.02.2021).

Merton, Robert K. 1942. "A Note on Science and Democracy", in Journal of Legal and Political Sociology 1, S. 115-126.

Nichols, Tom 2017. The Death of Expertise. The Campaign Against Established Knowledge and Why it Matters. Oxford: Oxford University Press.

Nolte, Paul 2020. «Widerwille und Trotz werden zunehmen - Corona als Gefahr für die Demokratie?", Interview in Frankfurter Rundschau, 29.03.2020.

Oreskes, Naomi; Conway, Erik M. 2010. Merchants of Doubt. How a Handful of Scientists Obscured the Truth on Issues from Tobacco Smoke to Global Warming. New York, Berlin, London: Bloomsbury.

Pfadenhauer, Michaela 2020. «Die Haltung ist doch: Die Schaubühne darf nicht sterben, das Berghain schon", Interview in Berliner Zeitung, 01.09.2020.

Pielke, Roger A. 2007. The Honest Broker. Making Sense of Science Policy and Politics. Cambridge: Cambridge University Press.

Pollmann, Arnd 2020. «Die neue Sehnsucht nach der Expertokratie», in Deutschlandfunk Kultur, 10.05.2020.

Rehrmann, Marc-Oliver 2020. Corona-Podcast: Auch Drosten für harten Weihnachts-Lockdown. https://www.ndr.de/nachrichten/info/Corona-Podcast-Auch-Drosten-fuer-harten-W eihnachts-Lockdown,coronavirusupdate140.html (Zugriff vom 03.02.2021).

Scharpf, Fritz W. 1999. Regieren in Europa: effektiv und demokratisch. Frankfurt/Main, New York: Campus. 
Strohschneider, Peter 2020. Zumutungen. Wissenschaft in Zeiten von Populismus, Moralisierung und Szientokratie. Hamburg: Kursbuch.

Weingart, Peter 2001. Die Stunde der Wabrheit? Zum Verhältnis der Wissenschaft zu Politik, Wirtschaft und Medien in der Wissensgesellschaft Weilerswist: Velbrück.

Leviathan, 49. Jg., Sonderband 38/2021 
Zusammenfassung: Wissenschaftliche Expertise ist in Krisen besonders gefragt. Ihre Einbindung in die Krisenbewältigung divergiert aber von Fall zu Fall, wie eine vergleichende Analyse der Klima-, Euro- und Coronakrise zeigt. Das betrifft sowohl den politischen als auch den medialen Einsatz von Experten. Überlagert werden diese Differenzen von einer Totalisierung der Expertenrolle, die durch die Kumulation bisher unvereinbarer Rollen zustande kommt und eine demokratisch produktive Funktion von Expertise erschwert.

Stichworte: Wissenschaftliche Expertise, Politikberatung, Krisenpolitik, Legitimation, Populismus, Medien

\title{
Expertise in Crisis: On the Totalisation of the Expert Role in the Euro, Climate and Corona Crises
}

Summary: Scientific expertise is particularly in demand in crises. However, its involvement in crisis response diverges from case to case, as a comparative analysis of the climate, euro and corona crises shows. This applies to the use of experts both in politics and the media. These differences are obscured by a totalisation of the expert role, which comes about through the accumulation of previously incompatible roles and makes a democratically productive function of expertise more difficult.

Keywords: Scientific expertise, policy advice, crisis politics, legitimacy, populism, media

\author{
Autor: \\ Prof. Dr. Caspar Hirschi, \\ Lehrstuhl für Allgemeine Geschichte, \\ Universität St.Gallen, \\ Müller-Friedberg-Strasse 6, \\ 9000 St.Gallen, \\ Schweiz
}

